\title{
Acción colectiva y activación del capital socio-territorial como reacción a la globalización: El caso de Montréal' 2
}

Juan-Luis Klein, Jean-Marc Fontan, Diane-Gabrielle Tremblay Universidad de Québec en Montréal

\section{Resumen}

Este trabajo constituye un estudio local visto desde una perspectiva global. El objetivo del estudio es indagar en el papel del territorio, en la reconversión económica y particularmente en la innovación social. Para ello, se consideran tres barrios peri-centrales de la ciudad de Montréal que dan una imagen de la dinámica social en donde los actores económicos y sociales se movilizan a fin de revitalizar el medio local.

Estos barrios peri-centrales han sido afectados por procesos de degradación urbana desencadenados por la desindustrialización, delocalización industrial y terciarización creciente. En términos sociales se pueden caracterizar por la exclusión social y desde una parte de

continúa

\footnotetext{
${ }^{1}$ Una versión preliminar de este texto ha sido presentada en inglés en el coloquio anual de la "Asociación para los Estudios Canadienses en los Países de Habla Germana", sobre el tema "Ciudad-Suburbios-Metrópolis", desarrollado en Grainau, Baviera, Alemania, del 20 al 22 de febrero de 1998.
}

${ }^{2}$ Traducción realizada por Alicia Lindón y revisada por Juan-Luis Klein. 
la teoría regional actual se podrían identificar como "regiones que pierden". Sin embargo, el surgimiento de redes asociativas en las comunidades locales parece estar revirtiendo los anteriores rasgos hacia formas de revitalización social.

De esta forma, el trabajo se integra de cuatro apartados. En el primero, se presenta la situación de la aglomeración de Montréal, comparandolascondiciones de los suburbios con la de los barrios peri-centrales. En segundo lugar, se considera el vínculo entre innovación y colectividad, destacando los elementos que conducen a lo que se ha denominado "medios innovadores". En tercer lugar, se analizan tres ejemplos que dan una imagen de la dinámica social de los barrios peri-centrales de Montréal, en donde los actores económicos y sociales se movilizan a fin de revitalizar el medio local. Finalmente, se propone un modelo que establece el vínculo entre la movilización social y la innovación, postulando que la movilización social es una condición para la innovación y la reconversión económica de espacios urbanos o regionales que se enfrentan a la degradación urbana.

Este trabajo se inserta en un análisis de tipo global sobre el papel que juegan los factores territoriales en la reconversión económica y, particularmente, en los procesos que conducen a la innovación social. Pondremos énfasis en el caso específico de Montréal, pero nuestra aproximación es global. Abordamos los proyectos de reconversión de ciertos barrios industriales que se han vuelto obsoletos a causa del proceso de desindustrialización y terciarización en curso desde los años ochenta. Estos barrios corresponden a lo que comúnmente ha sido denominado "regiones que pierden", en relación a las llamadas "regiones que ganan" (Benko y Lipietz, 1992). Sin embargo, es posible observar un nuevo dinamismo en ellos, en donde un número significativo de proyectos de envergadura diversa emergen, señalando la aparición de una nueva cultura de desarrollo de tipo asociativo (Hamel, 1991). En esta nueva cultura, las contradicciones de clases se matizan por la importancia que toma la pertenencia geográfica, lo que conduce a la emergencia de una conciencia territorial en los actores socioeconómicos (Klein, 1997).
Las explicaciones relativas a los factores que hacen ganar a ciertas regiones, mientras que otras parecen "perder la partida" no permiten explicar esta nueva vitalidad. Teñidas por el fatalismo, estas explicaciones le otorgan una importancia demasiado grande a las fuerzas del mercado, dejando poco campo a la acción colectiva destinada a contrarrestar a estas fuerzas. Es más, estas explicaciones omiten las actividades de naturaleza social y cultural, que por cierto no son directamente productivas pero que constituyen un ingrediente esencial para la reconversión de zonas en proceso de degradación (Bassand, 1990; Moulaert et al, 1994).

Nuestro trabajo está compuesto de cuatro partes. Primero, examinaremos rápidamente la situación de la aglomeración de Montréal, comparando la situación de los suburbios con la de los barrios peri-centrales e insistiendo sobre el "efecto de corona" ${ }^{3}$ que orienta la evolución de la aglomeración. En segundo lugar, nos referiremos rápidamente al vínculo entre innovación y colectividad, destacando los elementos importantes que constituyen lo que se ha convenido en denominar "medios innovadores". En tercer lugar, examinamos tres ejemplos que nos dan una imagen de la dinámica social de los barrios peri-centrales de Montréal, en donde los actores económicos y sociales se movilizan a fin de revitalizar el medio local. Por último, a título de conclusión, proponemos un modelo que establece el lazo entre la movilización social y la innovación, postulando que la movilización social es una condición para la innovación y la reconversión económica de espacios urbanos o regionales que se enfrentan a la degradación demográfica y económica.

\section{Montréal frente a la crisis: del efecto de corona a la movilización social}

La literatura sobre la problemática del desarrollo de Montréal es cada vez más abundante (Remiggi y Sénécal, 1992; Tellier, 1996; Bryant y Manzagol, 1998). De esta literatura se desprende que Montréal está pasando por un proceso de degradación que se explica por tres tipos de factores:

${ }^{3} \mathrm{NdT}$ : El efecto de corona hace referencia al proceso urbano según el cual las zonas centrales pierden población y actividades económicas, y las periféricas a ellas se van ocupando progresivamente. En Québec se habla del «effet de beigne», que en francés quebequens significa effet «donut», para designar a esta tendencia. 
1. El primero tiene que ver con la situación de Montréal en el conjunto norteamericano. La aglomeración de Montréal es parte de la gran región industrial del Noreste del continente. Y como parte integrante de lo que se ha conocido como "El cinturón industrial" (Manufacturing Belt), generado por la revolución industrial del siglo XIX, Montréal está experimentando los efectos del desplazamiento del centro de gravedad de la economía continental hacia el Sudoeste, hacia lo que se conoce como el Sun Belt (Thibodeau, 1996).

2. El segundo tipo de factores tiene que ver con la situación global en curso en Canadá, cuya economía no logra igualar el ritmo de crecimiento de su vecino del Sur, siendo aún más grande el retraso en el caso de la provincia de Québec (D.G. Tremblay, 1995). En 1995, las principales metrópolis canadienses, es decir, Toronto, Vancouver y Montréal, cuyas tasas respectivas de desempleo eran de $\mathbf{8 . 3 \%}$, $8.5 \%$ y $11.3 \%$, se situaban en la cola de la lista de las aglomeraciones norteamericanas (Trottier, 1996), lo que muestra las dificultades de la economía canadiense en general y de Montréal en particular.

3. El tercer tipo de factores tiene que ver con el cambio de papel que Montréal mantiene en el marco canadiense. La aglomeración ha perdido su rol de metrópolis económica nacional en beneficio de Toronto y su crecimiento parece estar limitado, cada vez más, al rol de metrópolis institucional y económica de la provincia de Québec (Sénécal y Manzagol, 1993; Proulx, 1997). De esta manera, su dinamismo depende, con mayor frecuencia, de la afirmación económica y política de la sociedad quebequense y del reforzamiento de sus interrelaciones con el resto de la economía de la provincia.

Estos tres órdenes de factores tienen un efecto sobre la situación económica interna de la aglomeración y sobre las estrategias que los actores sociales y económicos establecen con respecto a su reconversión. Las inversiones se desplazan, provocando, por una parte, el proceso de degradación de ciertos barrios, sobre todo de los barrios peri-centrales, situados en los alrededores del centro de la ciudad (Klein, Fontan, Tremblay y Tardif, 1998). Por otra parte, se observa el crecimiento de nuevas zonas productivas y residenciales en las afueras. Esto constituye lo que ha sido denominado el "efecto de corona". Esta divergencia entre las dificultades económicas del centro de la ciudad y el crecimiento económico y demográfico de los suburbios se ha intensificado, en el caso de Montréal ha sido a causa de la concurrencia intermunicipal y de la ausencia de un marco de concertación global y eficaz.

La degradación de los barrios del centro de la ciudad, sobre todo de los barrios peri-centrales, es notable a simple vista: los te- rrenos vacantes, las bajas importantes en el valor comercial de los inmuebles, las tasas elevadas de desocupación de los departamentos y de los espacios para oficinas, así como la disminución gradual de las arterias comerciales, son algunos de los signos de los efectos de una crisis que se prolonga y que se prolonga más en Montréal que en otras ciudades norteamericanas. Por esto se dice que Montréal se ha transformado en la capital canadiense del desempleo, de la pobreza y la exclusión.

Sin embargo, un examen más profundo nos muestra los signos de un proceso de reconversión y deja ver ciertos signos alentadores. En cuanto a la creación de empleos y de inversiones, se destaca el dinamismo de ciertos sectores económicos orientados hacia la exportación, tales como el aeronáutico, el farmacéutico, el del material de transporte y el de las telecomunicaciones. Claro que estos sectores están presentes sobre todo en los suburbios, explicando en parte el crecimiento de éstos. Por lo que respecta a la ciudad central, es necesario subrayar la existencia de una sólida red de instituciones y servicios de nivel superior, así como de una calidad de vida bastante excepcional para América del Norte (Coffey y Drolet, 1994). En cuanto a esto, debemos agregar la estructuración progresiva de un medio comunitario que se involucra cada vez más en el desarrollo económico (Hamel, 1995; Morin, 1996).

Organizaciones de orden diverso, representativas de los medios comunitarios, se forman en los barrios peri-centrales con el objetivo explícito de contrarrestar las fuerzas del mercado, responsables del deterioro de su medio de vida, y de asegurar una vitalidad social susceptible de llegar a ser un factor de desarrollo. Ciertamente, la expansión del medio asociativo está muy extendida en los países occidentales en donde diversas asociaciones, subordinadas al gobierno, asumen una parte de los servicios dejados vacantes por el repliegue del Estado, a causa del empuje del neoliberalismo y de la consecuente privatización de los programas de tipo social. Sin embargo, el fenómeno del que hablamos aquí es diferente.

En este caso, se trata más bien de una transformación más completa de los diferentes tipos de movimientos sociales, desde el movimiento sindical hasta el movimiento comunitario, los que tienden a tomar una parte activa, económicamente hablando, en el desarrollo (Hamel, 1997; Morin, 1996). Los barrios peri-centrales de Montréal han sido la cuna de esta nueva orientación de los movimientos sociales (Hamel, 1991; Fontan, 1991; Fabvreau y Ninacs, 1993). Así, estamos ante la presencia de un nuevo tipo de movimiento social que combina la acción colectiva y la inversión económica, 
movilizando las fuerzas locales de cara al desarrollo (Fitzgerald, 1991; Klein, Tremblay y Dionne, 1997).

La emergencia de este tipo de movimiento nos obliga a revisar las explicaciones de las tendencias que afectan al desarrollo local y regional. Varios autores han intentado explicar las diferencias entre los antiguos espacios industriales que decaen y el auge de nuevos espacios productivos, utilizando un modelo que combina la dualidad "crecimiento/decadencia" con otra dualidad espacial entre "regiones que ganan/regiones que pierden" (Benko y Lipietz, 1992). Este modelo traduce muy bien las características globales impulsadas por las fuerzas del mercado, que bajo el efecto de la globalización, favorecen la desindustrialización y la reestructuración de las aglomeraciones urbanas. Sin embargo, éste debe ser refinado a fin de tomar en cuenta la reacción de las colectividades locales que habitan las regiones denominadas "perdedoras" y que apuntan a invertir las tendencias impulsadas por las fuerzas del mercado (Moulaert, Delvainquière, Delladetsima, 1997).

El potencial que representa este tipo de reacción debe observarse a la luz de las nuevas teorías del desarrollo, centradas en la importancia del medio y en el papel primordial que juega la existencia de actores sociales dinámicos e innovadores en la estructuración de éste (Vachon, 1993; Tremblay y Fontan, 1994). Interroguémonos, pues, sobre lo que es un medio y sobre lo que aporta al desarrollo económico, particularmente en lo que respecta a la creación de empresas. La síntesis de los elementos conceptuales elaborados por los diversos autores que contribuyen a la explicación de lo que se denomina "los medios innovadores" nos permitirá responder este interrogante.

\section{El medio y la innovación social: la importancia de los actores y la conciencia territorial}

El concepto de "medio innovador" ha sido desarrollado con el fin de explicar las condiciones favorables al nacimiento de la empresa y la adopción de la innovación. Formulado en un principio por Aydalot (1984) y profundizado enseguida por diversos equipos europeos, este concepto postula que la empresa no pre-existe a los medios innovadores, sino que es el producto de éstos. La empresa es el resultado, y no la causa, de la capacidad de invención del medio, de sus capacidades para engendrar la innovación social y las innovaciones tecnológicas (Aydalot, 1986)
Esta visión converge con otra perspectiva, la de los "distritos industriales". Es Becattini quien, diciendo que el tipo de desarrollo observable en lo que se ha denominado "Tercera Italia" se ha caracterizado por la difusión y la multiplicación de pequeñas y medianas empresas que mantienen relaciones de cooperación y competencia, establece un lazo con los distritos industriales evocados por Alfred Marshall. Así, este autor pone la primera piedra de una explicación que Piore y Sabel (1984) contribuyeron a difundir en el seno de la comunidad científica. La noción de distrito industrial acentúa la importancia del potencial de desarrollo endógeno de una colectividad, en donde la población y las empresas se complementan (Becattini, 1992), potencial que desemboca en actividades de cooperación y de asociación entre los actores sociales y económicos de origen público y privado (Klein, 1992). A los vínculos entre las empresas, se agregan las relaciones sistemáticas entre ellas y las instituciones locales, lo que contribuye a la puesta en práctica de modalidades locales de regulación, frecuentemente tácitas, designadas como "convenciones" por interpretaciones más recientes (Storper, 1997).

Distrito industrial y medio innovador son dos perspectivas que explican lo que el medio aporta a la empresa y al desarrollo económico. En principio, es gracias a la organización de interdependencias mico. En principio, es gracias a la organizacion de interdependencias grado de incertidumbre de la inversión y de los empresarios. Es entonces, la colaboración productiva lo que les permite a las pequeñas y medianas empresas gozar de economías de escala, equivalentes a las de la gran empresa, haciendo posible una mayor flexibilidad. Por otra parte, estas interrelaciones favorecen el aprendizaje de una cultura técnica y productiva. Siendo, el medio el proveedor de instancias organizacionales que permiten a las empresas locales insertarse en las redes de la economía mundializada (Maillat, 1992; Quévit, 1992).

El medio es, pues, el resultado de un sistema de actores, de estructuras y de instituciones en interacción. Las prácticas organizacionales, la manera de utilizar las técnicas, de aprehender el mercado, el saber-hacer y la cultura ocupacional, el tejido institucional y el respeto de ciertas convenciones por parte de los actores, son elementos que caracterizan a un medio y que determinan su capacidad de producir la innovación. Su estructuración resulta de un conjunto de relaciones formales e informales, explícitas y tácitas, entre empresas y su entorno, cuya naturaleza es tanto cultural como productiva. Mucho más que la simple yuxtaposición de unidades de producción, el medio corresponde a un conjunto de vínculos entre las instancias políticas, sociales y económicas de una colectividad (Crevoisier, 1994; 
Storper 1997), conjunto de vínculos que permite una regulación local y flexible de las relaciones sociales (Klein, 1992).

Esta perspectiva hace intervenir la noción de territorio, que emerge como un factor fundamental del desarrollo. Debido a que si los actores se vinculan entre sí, ello es, esencialmente, porque comparten el mismo territorio y porque esto genera un sentimiento común de identidad provocando un proceso cognitivo colectivo que engendra dinamismos sociales, políticos, culturales, tecnológicos, administrativos y económicos (Becattini y Rullani, 1995). El territorio es a la vez el resultado y el marco de la reproducción de las relaciones entre los actores, las instituciones y las estructuras.

\section{Los barrios peri-centrales en Montréal: movilización comunitaria e innovación social}

Tal como lo hemos visto más anteriormente, dos tendencias han caracterizado a la aglomeración de Montréal durante los últimos decenios. Por una parte, hace frente a un proceso de desindustrialización en beneficio de diversas categorías de servicios, que proveen las tres cuartas partes del empleo (Coffey y Drolet, 1994). Por otro lado, se ha dado el despliegue de la ciudad a través de varias coronas de suburbios. Este doble movimiento está ligado a una serie de cambios en el modo de vida de los ciudadanos, así como a la aparición de nuevas actividades productivas cuyas exigencias son satisfechas más fácilmente en la periferia que en el centro. Los grandes perdedores de estos desplazamientos económicos y demográficos sonlos barrios pericentrales (Figura 1). Aunque estos barrios aún están habitados por una parte significativa de la población de Montréal, atraviesan por una aguda crisis demográfica. Entre 1981 y 1996, la población de éstos ha disminuido de manera importante, prolongando un proceso iniciado en los años setenta. En conjunto, ellos han perdido casi $10 \%$ de su población. Este descenso es aún más significativo si se considera que la población del conjunto de la aglomeración ha aumentado en un porcentaje equivalente.

La extensión del hábitat urbano no constituye un fenómeno nuevo en Montréal. Al contrario, en buena medida, es el efecto de una economía productiva que, a través de periodos muy distintos, siempre ha estado ligada a la evolución de las vías de transporte. En un primer periodo (desde la segunda mitad del siglo XIX hasta la Segunda Guerra Mundial), la construcción de las grandes infraestructuras del transporte ferroviario y portuario le dieron a la ciudad poderosos factores de localización industrial y residencial, lo que le permitió enarbolar el título de principal metrópolis económica canadiense hasta los años sesenta. Luego, los ferrocarriles decayeron en beneficio de las carreteras y del transporte por camión, esto tuvo un efecto importante sobre el dinamismo de los barrios industriales de antaño y abrió el camino para el desarrollo de nuevos sectores, tales como el del automovilístico, el de la aeronáutica y el de la industria farmacéutica, que se ubicaron progresivamente en las afueras. Paralelamente, la ciudad central, sobre todo los barrios peri-centrales, fue afectada por un proceso de "desvitalización económica" y de "desintegración social" que la hacía particularmente sensible a los efectos de las recesiones y de las crisis económicas.

Además de ser presa de la pérdida de vitalidad demográfica, estos barrios están habitados por una población que conoce los importantes problemas económicos, consecuencia de la situación global que reina en Québec (D.G. Tremblay, 1995), aunque en porcentajes muy superiores. El número de empleos ha disminuido, lo que no deja de afectar los ingresos de los habitantes. El porcentaje de población pobre (ingreso inferior a 10,000 dólares canadienses por año) es más fuerte aquí que en el conjunto. El porcentaje de familias monoparentales es superior al $30 \%$, superando en ciertos casos el $40 \%$, mientras que en el conjunto de la aglomeración este porcentaje es inferior al 25\%. Los débiles ingresos y la pérdida de empleo engendran dificultades sociales considerables que desembocan en la exclusión.

La gravedad de los problemas que afectan a los barrios pericentrales no ha tardado en generar una respuesta de los medios políticos y sociales. Por una parte, a partir de los años setenta, la administración municipal de Montréal, con el acuerdo de las otras instancias de gobierno, ha estado llevando a cabo una serie de proyectos destinados a atraer nuevos habitantes y nuevas inversiones, sobre todo en el terreno comercial. Por otra, la población residente se moviliza y aporta un nuevo dinamismo social a estos barrios, exigiendo del Estado los recursos necesarios para asegurar su revitalización. Uno de los principales resultados de estas movilizaciones reside en la creación de las Corporaciones de Desarrollo Económico Comunitario (CDEC) (Fontan, 1991; Morin, Latendresse y Parazelli, 1994). Al mismo tiempo, se crean numerosos grupos y asociaciones de tipo más sectorial y limitado con el objeto de favorecer el desarrollo local.

Es esta importante red asociativa lo que constituye la base de una economía social, cuya tendencia muy bien podría influir en el conjunto de la economía de Québec (Fontan, 1997; Guay, 1997). A fin de ilustrar la importancia de esta realidad, examinamos tres ejemplos. 
El primero corresponde a las $\mathrm{CDEC}$, lo que presentamos como una suerte de institucionalización de la movilización social, en relación aunque también en contradicción, con el Estado. El segundo ejemplo corresponde a la Sociedad de Desarrollo Angus, que constituye un caso de implicación del medio comunitario en la reconversión voluntaria de un barrio importante de Montréal. Y el tercero, se refiere al Barrio de los Recoletos (Faubourgdes Récollets), un barrio en donde la movilización social se combina con la implantación de un nuevo tipo de empresas privadas, facilitando así la reconversión, más o menos espontánea, de uno de los barrios más afectados por la desindustrialización. Se trata de tres aspectos, de tres tipos de procesos, de tres escalas, a través de los cuales se estructuran medios susceptibles de crear innovaciones sociales.

\section{Las corporaciones de desarrollo económico y comunitario (CDEC):} semilleros de innovación social

Luego de una primera etapa de experimentación en ciertos barrios (Pointe-Saint Charles, Centre-Sud y Hochelaga-Maisonneuve), las CDEC han llegado a ser una estructura de concertación generalizada en el conjunto de la ciudad de Montréal, operando en la escala del arrondissement. ${ }^{4}$ Las modalidades de intervención de estas CDEC difieren según las características del medio y de su anclaje social. En todos los barrios, pero sobre todo en su cuna, es decir en los barrios peri-centrales, las CDEC se transforman en los actores ineludibles del desarrollo económico (Favreau y Ninacs, 1993). Las CDEC se constituyen en interlocutores representativos del medio (Morin, 1995) representando a todas las capas sociales que forman parte de sus órganos directivos. Por cierto, esto no significa que la situación sea homogénea (Cuadro 1).

La principal característica de las CDEC es que trabajan en nombre de toda una comunidad local y no solamente de sus miembros

4. Los arrondissement son las unidades administrativas infra-municipales creadas por la ciudad de Montréal, de las cuales el arrondissement Ville-Marie corresponde al centro de la ciudad. Cinco de estos arrondissement corresponden a lo que estamos denominando barrios pericentrales. Siete $\mathrm{CDEC}$ han sido implantados en el territorio de Montréal en los arrondissements. Esta ecuación CDEC-arrondissements no siempre ha respetado la especificidad de los medios, y en ciertos casos, ha reunido a colectividades que no comparten el mismo sentimiento de identidad, lo que a veces ha creado problemas e impuesto compromisos (Morin, 1997). NdT. Los arrondissements corresponden más o menos a lo que en la ciudad de México son las "delegaciones" en tanto unidades administrativas, sin embargo por la escala territorial se podrían asemejar a las "colonias"

(Favreau y Ninacs, 1993). Es así que, como consecuencia de los graves problemas económicos que atraviesan las comunidades que ellas representan, su primera misión, común a todas las $\mathrm{CDEC}$, reside en la defensa de los trabajadores afectados por la deslocalización económica y en su reinserción en el mercado de trabajo. Esto explica que sus primeras acciones hayan tomado la forma de proyectos de creación de empleo y de formación, financiados por subvenciones municipales y gubernamentales. Sin embargo,progresivamente, estas corporaciones adoptan estrategias más elaboradas, penetrando en el universo de las inversiones, asociadas a su vez con las instituciones y empresas locales privadas (Fontan, 1994; Favreau, 1995).

Las CDEC son financiadas por diversas instituciones, gubernamentales y municipales, como la ciudad de Montréal, la Secretaría de Asuntos Regionales, el Ministerio de la Industria, Comercio, Ciencias y Tecnología de Québec, la Banca Federal y de Desarrollo, el Ministerio de Desarrollo de los Recursos Humanos y la Sociedad de Québec de Desarrollo de la mano de obra. Sus acciones, múltiples, se inscriben en tres grandes ejes: la concertación, el empleo y los servicios a las empresas.

La concertación es uno de los objetivos estratégicos de las CDEC. La colaboración de los diferentes tipos de actores y la negociación local de sus intereses constituyen, sin duda, un primer paso hacia la movilización de las fuerzas locales en función de la revitalización de su colectividad. En efecto, la concertación permite a los actores ponerse en relación y descubrir sus objetivos comunes. Aunque ésta sea inherente al conjunto de las acciones de las $\operatorname{CDEC~(han~sido~creadas~}$ para ello), ciertos proyectos hacen de ella un objetivo específico; más aún cuando ciertas CDEC se han asociado creando redes interarrondissements e inter-institucionales.

La segunda gran misión de las CDEC tiene que ver con la reinserción de los desempleados en el mercado de trabajo. En estos barrios, devastados por el desempleo y por el cierre de empresas, esta misión se ha traducido en proyectos orientados hacia la recalificación de los individuos, para que éstos se puedan reintegrar en un mercado de trabajo en reestructuración acelerada. Las CDEC ofrecen servicios de información, de apoyo y de referencia sobre las oportunidades de empleo. Además, organizan actividades de capacitación de personas sin empleo. El intercambio sistemático de información entre las $\mathrm{CDEC}$, en relación a la oferta de empleos, permite la creación de una base de información muy extensa. Así, las personas deseosas de reintegrarse al mercado de trabajo pueden disponer de una red de in- 
formación, que es reconocida como primordial en la búsqueda de empleo.

Los proyectos de capacitación oformación para el empleo que orientan la reinserción de los desempleados en el mercado de trabajo, generalmente se ofrecen en colaboración con los organismos locales sin fines de lucro, y son subvencionados gracias a organismos gubernamentales. De esta manera las CDEC aportan un complemento a los servicios dados por las instituciones públicas y ayudan a vincular los programas de capacitación a las necesidades de la comunidad local en materia de la búsqueda de empleo.

La tercera misión de las CDEC es el apoyo al empresariado y a la creación de empresas. Aunque crucial, este tipo de intervención se revela más compleja y la experimentación aún está en curso. Puede señalarse sin embargo, que un primer tipo de servicio ofrecido por las $\mathrm{CDEC}$ a las empresas se refiere a la información y la capacitación. Cabe señalar que, ciertas CDEC también aseguran la ayuda financiera a las empresas locales, con el apoyo de fondos públicos descentralizados. Una encuesta reciente muestra que las CDEC tienen una cierta influencia sobre el empresariado local, aunque la fuerza de esta influencia y los efectos de ella no han sido evaluados de manera convincente (Morin, 1996).

Es importante subrayar también, que ciertas CDEC exploran vías estratégicas susceptibles de atraer inversiones económicas mayores y de canalizar colaboraciones locales que puedan ser suscitadas por estas inversiones. Por supuesto, este tipo de proyectos es difícil de poner en marcha, ya que exigen un nivel de concertación que desborda el marco de acción de una CDEC, necesitando además recursos financieros importantes. Sin embargo, tratándose de proyectos estructurantes (que podrían tener consecuencias importantes sobre el conjunto de la economía de Montréal), este aspecto merece un análisis más detallado. Uno de estos proyectos es el del Tecnopolo ambiental Angus en el barrio de Rosemont, que analizaremos con más detalles a continuación.

\section{El tecnopolo ambiental Angus: el medio comunitario se lanza a los negocios}

Desde hace cinco años, el barrio Rosemont, uno de los más antiguos barrios industriales de Montréal, se ha transformado en el teatro de un importante proyecto de reconversión económica. Este proyecto trata de la utilización de un terreno dejado vacante por el cierre de una importante fábrica, los Talleres Angus (de ahora en adelante: Angus), cuyo objetivo principal es el de crear un parque industrial que pueda actuar como locomotora del desarrollo de toda la zona. Es, pues, una experiencia voluntaria de reconversión, aunque, diferente de las experiencias voluntarias clásicas, en donde el principal instigador y actor es el gobierno. En este caso, el instigador y principal actor del proyecto es una organización del medio, creada por la colectividad local. Se trata de la Sociedad de Desarrollo Angus (SDA), que reúne a los principales actores sociales del barrio y que ha sido organizada por la CDEC de Rosemont-Petite Patrie (Fontan y Yaccarini, 1996).

Angus pertenecía a la compañía Canadian Pacific Rail (de ahora en adelante: CPRail), uno de los grandes boldings canadiense que opera en el transporte ferroviario. Esta fábrica constituía la principal empresa de un barrio cuyo conjunto, por su presencia, había contribuido a construir. Su cierre constituyó el desenlace de la decadencia de las actividades ferroviarias de CPRail en Montréal y del desmantelamiento progresivo de su vía férrea, vía que por otro lado, había estructurado lo que fue, a inicios del siglo XX, uno de los principales corredores industriales de la ciudad. Aunque la vía todavía sea utilizada y aunque varias industrias estén también activas (el corredor emplea a unas 20,000 personas, sobre todo en el sector textil), numerosos equipamientos, tales como los patios de maniobras, ${ }^{5}$ los talleres de reparaciones y las vías secundarias han llegado a ser inútiles. Las infraestructuras han sido desmanteladas, o están a punto de serlo, y numerosas fábricas han abandonado la zona, dejando el sector residencial, sobre todo las partes más próximas a la vía, en un estado de desintegración.

Angus producía y reparaba locomotoras y vagones para ferrocarriles, dando empleo a un número de trabajadores que variaba entre 2,000 y 7,000 según los periodos. ${ }^{6}$ Desde su fundación en 1904 , esta empresa fue innovadora, en el plano de la tecnología y de la gestión de la fuerza de trabajo. Típicamente fordista, fue una las primeras de Montréal en asociar el taylorismo, el trabajo en cadena, los salarios elevados, e incluso, ciertas ventajas sociales; éstas fueron conquistadas paulatinamente luego de conflictos de trabajo épicos.

${ }^{5} \mathrm{NdT}$ : Estamos traduciendo "cours de triage" como patio de maniobra, lo que hace referencia a los equipamientos ferroviarios en donde se realiza la selección y el reagrupamiento de los vagones para formar los convoyes.

${ }^{6}$ En su momentos culminante, durante la Segunda Guerra Mundial, este complejo ocupaba 12,000 personas en la fabricación de material de guerra. 
Muy productiva y muy eficiente durante la primera mitad del siglo, la empresa comenzó a decaer durante los años sesenta. En 1974, mientras se iniciaba el desmantelamiento de las diferentes unidades productivas, la fábrica empleaba apenas 1,000 personas. Este desmantelamiento se continuó hasta enero de 1992, momento de su cierre completo y definitivo.

El cierre de Angus planteó el problema de la reconversión de la zona y, por supuesto, del sitio en el cual la fábrica trabajaba. En un principio, ésta ocupaba un terreno de alrededor de un millón de metros cuadrados, lo que es una superficie considerable. Sin embargo, ya en 1974, el CP Rail había transferido la mitad del terreno a su filial Marathon, empresa dedicada a la gestión inmobiliaria, que propuso implantar un centro comercial de gran envergadura y un sector residencial destinado a una clientela de fuertes ingresos. En razón de las consecuencias que este proyecto hubiera tenido sobre el costo de los alquileres y sobre la estructura comercial de sus principales arterias, su presentación en 1976 desencadenó la oposición inmediata tanto de los residentes como de los comerciantes. Todos ellos se movilizaron contra el CP Rail para hacer abortar el proyecto, implicando al ayuntamiento. En 1982, después de más de seis años de presiones y como resultado de un consenso obtenido entre los representantes del medio local, el ayuntamiento y el gobierno, esta parte del terreno fue adquirida por una sociedad para-municipal, conviniéndose que el desarrollo inmobiliario del sitio debía contener $40 \%$ de viviendas sociales destinadas a personas de bajos ingresos.

El mismo problema volvió a plantearse en 1992 cuando el cierre de lo que quedaba de Angus dejó vacante la otra mitad del terreno. La controversia se vuelve a plantear entre los propietarios de la empresa y los actores locales, representados en esta ocasión por la CDEC local que, desde su creación en 1989, reagrupa a los representantes de diversos sectores socioeconómicos (comercio, sindicatos, medio asociativo, etcétera). La empresa CPRail, propietaria del sitio, reedita su proyecto anterior, promoviendo un desarrollo de tipo multifuncional basado en la construcción residencial y de grandes centros comerciales. En cuanto a los actores locales, representados por la CDEC, en lo que podría ser visto como un giro en sus objetivos, recomendaron mantener la vocación industrial del terreno en relación a sus antiguas reivindicaciones.

${ }^{7}$ Se trata de la Sociedad de Desarrollo de los Terrenos de los Talleres Angus (SOTAN).
La posición de la empresa implicaba una derogación en el reglamento de usos del suelo de la ciudad, ya que el sitio estaba destinado a un uso industrial. Para obtener esta derogación se necesitaba el acuerdo del ayuntamiento, quien por cierto, fue objeto de importantes presiones de parte de los actores locales. Frente a la evidencia del rechazo de su opción por el medio local y por el ayuntamiento, CP Rail emprendió negociaciones con la CDEC en lo relativo al futuro del sitio. La CDEC se transformó en la punta de lanza de una extensa movilización del medio local. Representados por la CDEC local, los actores sociales proponían el relanzamiento de la industria del sector, implantando un parque industrial sobre el sitio de las fábricas Angus. Este parque estaba destinado a ser, según el proyecto de la CDEC, el motor de un sistema productivo local (Cuadro 2).

En mayo de 1994, la CDEC recibe el mandato, luego de una asamblea pública, de adquirir el terreno. Esta vez no hubo una confrontación abierta con la empresa propietaria, como lo fue durante los episodios de los años setenta, sino negociaciones y juego de influencias. Se llegó a un acuerdo en septiembre de 1994, según el cual $C P$ Rail se compromete a vender la mitad del terreno a la CDEC por diez millones de dólares. Según este mismo acuerdo, la otra mitad sería consagrada al desarrollo residencial.

A partir de este momento, luego de una serie de estudios técnicos, se concluyó que el desarrollo de la parte industrial del terreno tomaría la forma de un parque de empresas especializadas en tecnologías ambientales, concebido a partir de los conceptos de distrito industrial y de medios innovadores, y que el proyecto sería dirigido poruna corporación independiente, representativa del medio: la Sociedad de Desarrollo Angus (de ahora en adelante: SDA). Para financiar el proyecto, la SDA hace un llamado a los principales actores económicos locales: las cajas populares, los bancos, Hydro-Québec, otras empresas y por supuesto, la CDEC.

Este proyecto se encuentra aún en una etapa de planificación. El parque todavía no es una realidad, por lo tanto es difícil evaluar los resultados. Sin embargo ya se están viendo algunos efectos. Además de asegurar el control del terreno, la SDA ha elaborado un plan estratégico de desarrollo que incluye un plan de ordenamiento del sitio, también ha emprendido diligencias dirigidas a promover el proyecto entre las empresas susceptibles de interesarse en las ventajas que ofrece el proyecto, tanto por el sitio como por el concepto.

Paralelamente, para asegurarse que la población local pueda sacar provecho de las repercusiones de éste, se ha creado un comité paralelo, cuya función es la de prever las medidas necesarias para la 
formación de mano de obra local. Compuesto por 19 miembros, representando una amplia gama de actores (medios comerciales, asociaciones, instituciones, medio sindical, etcétera), este comité ha elaborado un Plan Estratégico de adaptación de la mano de obra que estudia, entre otras cosas, el modo apropiado para asegurar la capacitación necesaria para desarrollar empresas de tipo ambiental. Estos trabajos han sido realizados con los organismos y las instituciones de la comunidad, lo que ha permitido un amplio consenso alrededor de sus proposiciones.

La construcción del parque se realizará por etapas, en un periodo de 10 años, siendo la primera la construcción de un complejo industrial multilocativo de un costo de diez millones de dólares. Ampliamente inspirado en los conceptos de distrito industrial y medio innovador, este proyecto se orienta tanto al desarrollo local como a la inserción del Tecnopolo en una red tecnológica de envergadura norteamericana. El Tecnopolo es mucho más que un parque industrial puesto que pone el acento en la sinergía entre actores del medio local e incluso del conjunto de la ciudad de Montréal. Así, la resistencia de los actores locales ante un proyecto que, según ellos, amenazaba sus intereses y su decisión de promover un plan mutiplicador, ha contribuido a la revitalización social del barrio, a la creación de instituciones representativas, a la elaboración de nuevos proyectos, en fin, a un conjunto de innovaciones sociales susceptibles de provocar el desarrollo económico.

La movilización comunitaria como complemento de la acción empresarial: el ejemplo del barrio de los Recoletos (Faubourg des

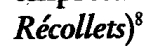

El Barrio de los Recoletos constituye otro caso de reconversión industrial, donde se combinan los aspectos espontáneos y voluntarios para que la ciudad de Montréal y el medio local se concerten alrededor de una estrategia de desarrollo. Como en el caso de Rosemont, este barrio es parte de la zona industrial que rodea al centro urbano. Más aún, fue la cuna de la industrialización canadiense y de Montréal. Sus raíces se remontan al año de 1830 . Sin embargo, es entre 1880 y 1930 que recibe las estructuras industriales ligadas a la construcción de las nuevas vías de transporte, tales como la vía férrea, que une el

${ }^{8}$ Esta parte se apoya en una actividad de síntesis realizada por Sylvain Provost presentada en 1997, a fin de obtener una maestría en geografía en la Universidad de Québec en Montréal. territorio canadiense y el Canal de Lachine, que prolonga la navegación fluvial hacia la región de los grandes lagos. En estos años, el barrio se erige como un importante barrio industrial. Las fundiciones, las industrias energéticas (electricidad) y las infraestructuras de almacenamiento (de cereales, frigoríficos, sal, carbón, etcétera) se desarrollan de manera espectacular, reemplazando a las actividades industriales artesanales y las construcciones residenciales de los inicios.

Los factores de localización de tipo portuario explican la emergencia y la decadencia de este barrio. Dependiente del puerto de Montréal y de las actividades de importación y exportación, sus actividades económicas eran tributarias de las crisis económicas, de las políticas macroeconómicas y de los cambios tecnológicos que fueron reduciendo progresivamente el rol del puerto de Montréal en el conjunto de la economía canadiense. Así es como a partir de la posguerra, el barrio atraviesa por una fase de degradación. Esta degradación se intensificó con la apertura de la vía marítima del río San Lorenzo, en 1959, y el consecuente cierre del Canal de Lachine algunos años más tarde.

Las empresas que se habían instalado allí atraídas por el puerto y por el canal, cierran o emigran y no son reemplazadas, dejando al y porrio en un estado de deterioro y desestructuración social completo. Esta situación se acentúa por la contaminación de los suelos, producida por más de un siglo de fuerte actividad industrial. Por otra parte, la construcción de ciertas infraestructuras de transporte, una vía férrea en 1933 y una autopista en 1965, separaron al barrio de su entorno inmediato, aislándolo de otras partes de la ciudad de las cuales el barrio formaba parte y tomaba su identidad. Luego de perder sus empresas y sus empleos, el barrio perdió su alma, su memoria y sus residentes.

En 1980, este barrio presentaba los rasgos de un lugar devastado, aún presentes en la actualidad a pesar de la aparición de signos evidentes de revitalización. Los edificios que subsisten son habitados por ocupantes ilegales y otros ceden el paso a terrenos de estacionamiento $(37.5 \%$ del suelo). La mayor parte del parque inmobiliario está en el abandono. Los servicios a los ciudadanos son inexistentes y la población residente está marcada por el signo de la marginalidad. Así, el barrio da la imagen de un verdadero no man's land (Sénécal et al, 1996).

Preocupados por esta situación, los funcionarios municipales decidieron actuar y reconvertir el barrio. Entre 1989 y 1992, la administración municipal adquirió una buena parte (más del $80 \%$ ) del 
parque inmobiliario (terrenos y construcciones), a fin de desarrollarlo y de atraer a una nueva capa de población. Con este fin, la administración municipal preveía la construcción de un sector residencial constituido por 2,000 unidades de habitación de alto nivel. Evidentemente, esto hubiera implicado la destrucción de las antiguas estructuras industriales y su reemplazo por una nueva morfología.

Esta idea no carecía completamente de sentido en sí, ya que la situación del barrio (próximo al centro de negocios y al casco antiguo de la ciudad), es atractiva, sobre todo para una población de altos ingresos, envejecida, que desea dejar los suburbios para regresar al centro. Sin embargo, el concepto se vuelve impracticable. Por una parte, en la aglomeración en su conjunto, y en la ciudad en particular, la oferta de unidades de vivienda sobrepasa la demanda, lo que hace que los promotores privados se interesen poco en este tipo de proyectos. Por otra, los nuevos modelos de arquitectura y culturales ya no tienden a la destrucción del patrimonio, sino que más bien a su protección.

Mientras tanto, la ciudad alquila a bajas tasas los edificios a artistas y artesanos que encuentran en estas instalaciones, la centralidad, viviendas baratas y el espacio de trabajo que les hacían falta. Estos artistas se sienten identificados con el barrio. Es por esto que se oponen al proyecto municipal, que entre otras cosas, implicaba su expulsión.

La necesidad de revisar el proyecto municipal se hizo evidente. Por lo que ahora, se elabora un nuevo proyecto, esta vez con el apoyo de los residentes. Este nuevo proyecto apunta a la renovación de los edificios con fines residenciales, para preservar la memoria histórica. Este hecho es significativo pues, curiosamente, esta memoria denominada histórica, en realidad está ligada a una identidad construida recientemente. El término Barrio de los Recoletos (Faubourg des Récollets), no se aplicaba en los inicios al territorio actual. Toda la parte Sur pertenecía a otro barrio, más grande, denominado "Griffintown", de donde fue aislada por infraestructuras de transporte que se transformaron en barreras (la autopista Buenaventura y la vía férrea). Los actuales residentes no tienen nada que ver con las antiguas actividades del barrio, lo que puede explicar la facilidad con la que ellos enarbolan una identidad de construcción social reciente. El hecho es que esta nueva identidad se revela movilizadora en relación a los actores locales y atractiva para ciertas empresas.

En efecto, paralelamente a la acción municipal, a partir del año 1985 , ciertas empresas comienzan a instalarse en el barrio. La combinación de lo antiguo y lo nuevo, de la modernidad y la posmo- dernidad que vehicula esta nueva identidad, se revela interesante para ciertas actividades económicas que asocian las artes, las nuevas tecnologías y los servicios. Aparte de algunas empresas de envergadura (se trata sobre todo de una miríada de pequeñas empresas, que asocian el arte y la tecnología y combinan la actividad industrial y los servicios de alto nivel, lo que configura una suerte de distrito industrial), más de la mitad de las empresas (56\%) se han implantado después de 1991 (Groupe Espace-Temps, 1997). Polarizadas por la industria de multimedia, en crecimiento fulgurante desde 1995, estas empresas se han ido instalando en las antiguas construcciones industriales, renovándolas, aunque conservando su cariz de antaño (Cuadro 3).

Por otro lado, la población del barrio, compuesta por artesanos, pequeños industriales de diversos dominios y artistas, se ha unido, constituyendo una corporación de desarrollo del tipo CDEC. Formado en 1997, este organismo busca llegar a ser el representante autorizado del barrio. Ampliamente representativo, este organismo se ha transformado en un actor ineludible en la transformación del barrio, animado por un profundo interés de revitalización (Sénécal et al, 1996).

De esta manera, la nueva dinámica del barrio ha cristalizado en una estrategia asociativa de desarrollo, en donde los actores principales son la ciudad y la población local y en la cual también participan otros actores socioeconómicos públicos y privados. Esta estrategia se apoya en dos principios que crean consenso: la mezcla de funciones (residencial, servicios, industrial) y la yuxtaposición de las artes y la tecnología.

Este proceso de reconversión está bastante adelantado. El barrio está tomando características que podrían transformarlo en un medio innovador. Las nuevas empresas que intervienen en dominios de alta tecnología y funcionan a escala norteamericana constituyen una masa crítica significativa, que podría actuar como un polo de atracción sobre otros establecimientos. Incluso, el barrio está cambiando progresivamente su imagen, presentando, poco a poco, connotaciones más positivas. La proximidad del casco antiguo de la ciudad y del puerto, sitios de intensa actividad recreativa y turística, así como del Canal de Lachine, en vías de revalorización con fines recreativos, deberían facilitar esta reconversión. El peligro, evidentemente, es que todo este proceso afecte negativamente a la población residente, como se ha visto en numerosos casos de gentrificación en América del Norte. El rol principal de la corporación local es, por lo tanto, el de velar por los intereses de la población local, lo que 
conseguirá más fácilmente participando en el proceso y no combatiéndolo.

\section{Capital socio-territorial y medios innovadores: pistas para una interpretación}

Recordemos que hemos planteado la hipótesis de que la vitalidad de los actores de un medio es esencial para la puesta en marcha de la innovación en el plano productivo. Los casos estudiados nos permiten entrever diversos indicadores de una cierta vitalidad institucional y social, aun en zonas que presentan características de colectividades en decadencia. Esta vitalidad corresponde a lo que denominamos "capital socio-territorial", que hemos definido como el conjunto de recursos humanos e institucionales que una colectividad puede movilizar a fin de acrecentar su potencial de desarrollo y permitir el bienestar de sus habitantes. El capital socio-territorial es el resultado de la amalgama de diversos componentes, tales como:

- valores compatibles, aunque no necesariamente homogéneos

- instituciones locales eficaces y adaptadas a los intereses de la población local

- instancias de negociación de los conflictos sociales a una escala local

- convenciones sólidas y bien arraigadas entre los actores sociales y las instituciones

- un medio asociativo activo y vigoroso

- una percepción positiva de la comunidad y de la pertenencia a ésta

- la difusión de la información

- instituciones financieras locales ancladas en el medio

Entendemos que la activación del capital socio-territorial depende de la identidad colectiva y de la conciencia territorial de los actores sociales que habitan y trabajan en una colectividad (Moulaert et al, 1997). Sin embargo, es necesario reconocer que la identidad y la conciencia territorial no se pueden establecer por decreto. Éstas se construyen socialmente, como lo demuestran los tres casos estudiados. Ellas son el resultado de acciones colectivas, de la movilización, y no de la tradición o de la historia, aunque éstas puedan tener importancia desde el punto de vista ideológico. En los casos estudiados, ellas son el resultado de acciones colectivas destinadas a contrarrestar las tendencias a la desestructuración provocadas por la globalización/delocalización (figura 2).

La movilización es una reacción a la desestructuración provocada por la crisis social y económica que resulta de la globalización y de los cambios que ella produce en cuanto a los factores de localización de las actividades productivas. Las acciones colectivas conducidas por los actores socioeconómicos y por los ciudadanos afectados por la delocalización de las industrias y las fuentes de empleo constituyen un factor de cohesión social. Estas acciones colectivas crean el medio forjando vínculos sociales, integrando a los grupos excluidos y creando una identidad colectiva.

Podemos concluir que las regiones que pierden pueden cambiar su destino. Los medios innovadores no son únicamente el producto de la empresa. Ciertamente, el desarrollo de medios locales innovadores necesita aportes exteriores. Sin embargo, creando las bases de una dinámica asociativa y actuando en el sentido contrario a las tendencias dominantes, la movilización social comunitaria se inserta en un proceso más extenso, que desborda los límites del desarrollo local de un barrio en particular. Por una parte, la movilización redefine los límites de lo que ha sido considerado como la economía de la eficiencia, creando puentes entre la acción social y la acción económica, así como también entre lo voluntario y la empresa financiera. Por otra, influye en el desarrollo de la aglomeración urbana en su conjunto, revitalizando el centro.

Evidentemente, el desarrollo económico de una gran metrópolis no puede limitarse a la acción comunitaria. Tampoco es obligatorio que toda acción comunitaria tenga que conducir al desarrollo económico. Sin embargo, ésta constituye un germen de desarrollo en el caso de las zonas y regiones que pierden como resultado de las crisis engendradas por las deslocalizaciones y la globalización. La teoría del desarrollo debería de tenerlo en cuenta y conceptualizarlo. 
Cuadro 1

Origen social de los miembros directivos de las corporaciones de desarrollo económico y comunitario (CDEC) de los barrios peri-centrales

\begin{tabular}{||l|c|c||}
\hline \multicolumn{1}{|c|}{ Medio de origen } & \multicolumn{2}{|c|}{ CDEC de los barrios peri-centrales } \\
\hline & $\begin{array}{c}\text { Participación } \\
\text { porcentual }\end{array}$ & Número \\
\hline $\begin{array}{l}\text { Grupos } \\
\text { comunitarios }\end{array}$ & 32,4 & 24 \\
\hline $\begin{array}{l}\text { Comercio y } \\
\text { finanzas }\end{array}$ & 29,7 & 22 \\
\hline $\begin{array}{l}\text { Instituciones } \\
\text { públicas y semi- } \\
\text { públicas }\end{array}$ & 13,5 & 10 \\
\hline $\begin{array}{l}\text { Organizaciones } \\
\text { sindicales }\end{array}$ & 8,1 & 6 \\
\hline Pobladores & 7,2 & 6 \\
\hline Empleados & 5,4 & 4 \\
\hline Otros & 2,7 & 2 \\
\hline Total & 100,0 & 74 \\
\hline
\end{tabular}

Cuadro 2

La posición de los actores en el debate sobre la reconversión de Angus

\begin{tabular}{|c|c|c|}
\hline $\begin{array}{l}\text { Actores } \\
\text { Posición }\end{array}$ & CPRail & CDEC \\
\hline Posición principal & $\begin{array}{l}\text { - desarrollo multifuncional } \\
\text { - construcción residencial } \\
\text {-implantación de centros } \\
\text { comerciales }\end{array}$ & $\begin{array}{l}\text { - desarrollo industrial } \\
\text { - Implantación de un polo } \\
\text { científico y tecnológico } \\
\text {-innovación }\end{array}$ \\
\hline $\begin{array}{l}\text { Posición c/respecto } \\
\text { al desarrollo } \\
\text { industrial }\end{array}$ & $\begin{array}{l}\text {-no es viable a causa de la } \\
\text { fuerza de atracción de los } \\
\text { barrios industriales } \\
\text { periféricos y de los suburbios } \\
\text {-Abundancia de espacios } \\
\text { industriales en Montréal }\end{array}$ & $\begin{array}{l}\text { - necesidad de crear empleo } \\
\text { durable } \\
\text {-detener el crecimiento } \\
\text { urbano desmedido } \\
\text { - hacer coincidir la creación } \\
\text { de empleo y las necesidades } \\
\text { locales }\end{array}$ \\
\hline $\begin{array}{l}\text { Posición c/respecto } \\
\text { al desarrollo } \\
\text { residencial }\end{array}$ & $\begin{array}{l}\text { - es lo más rentable } \\
\text {-aumentar el número de } \\
\text { viviendas disponibles } \\
\text { - situación geográfica ideal } \\
\text { con respecto a los } \\
\text { equipamientos urbanos } \\
\text { - mejoramiento de los } \\
\text { equipamientos recreativos } \\
\text {-integración del sitio en la } \\
\text { trama urbana }\end{array}$ & $\begin{array}{l}\text { - no crea empleo } \\
\text { - no tiene efectos } \\
\text { multiplicadores } \\
\text {-anteriormente, una parte } \\
\text { del terreno ya había sido } \\
\text { objeto de un importante } \\
\text { desarrollo residencial }\end{array}$ \\
\hline $\begin{array}{l}\text { Posición c/respecto } \\
\text { al desarrollo } \\
\text { comercial }\end{array}$ & $\begin{array}{l}\text {-consolidación de las } \\
\text { actividades comerciales de la } \\
\text { zona }\end{array}$ & $\begin{array}{l}\text {-destrucción del comercio } \\
\text { existente } \\
\text {-desvitalización de las } \\
\text { arterias comerciales }\end{array}$ \\
\hline
\end{tabular}


Cuadro 3

Establecimientos localizados en el Barrio de los Recoletos, según el año de implantación y el sector de actividad

\begin{tabular}{|c|c|c|c|c|c|}
\hline \multirow[t]{2}{*}{ SECTOK } & \multicolumn{4}{|c|}{ ANOO DE TMPLANTAGIÓN } & \multirow[t]{2}{*}{ TOIAL. } \\
\hline & Ariter de 1985 & 1985.90 & 1991.94 & Desputex de 1995 & \\
\hline \multicolumn{6}{|l|}{ ACIIVIDADES INNOYADAS } \\
\hline Imprenta y cdiciois & 4 & 5 & 7 & t) & 17 \\
\hline Comunicarusnes & 1 & 0 & 7 & 0 & 8 \\
\hline Mulitimledix & 0 & 2 & 4 & 10 & 16 \\
\hline Cientifieas y facticas & 1 & 2 & 4 & 3 & 12 \\
\hline Informática & 2 & 3 & 3 & 3 & 11 \\
\hline Orris & 0 & 5 & In & 22 & 45 \\
\hline Sub-total & 8 & 17 & 43 & 41 & 109 \\
\hline \multicolumn{6}{|c|}{ ACTIVIDADES TRADICIONALES } \\
\hline Inmotiliariss, financierai & 4 & 6 & 6 & 3. & 21 \\
\hline 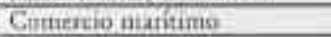 & 8 & 4 & 3 & 0 & 15 \\
\hline Comercio mayorina & 11 & 5 & 3 & h & 27 \\
\hline Transporte y almarenamiento & 4 & (1) & 6 & o & 23 \\
\hline Vestido & 0 & 0 & 4 & 4 & 8 \\
\hline Oras & 20 & 17 & 2) & 30 & 78 \\
\hline Sub-total & 49 & 45 & 43 & 35 & 172 \\
\hline \multicolumn{6}{|l|}{ SERVICIOS PUUIICOS } \\
\hline Oficinas del pobicino & 2 & 2 & 3 & 0 & 7 \\
\hline TOTAL & 59 & 64 & 89 & 76 & 258 \\
\hline
\end{tabular}

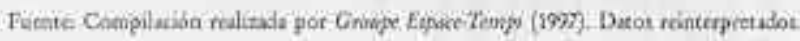

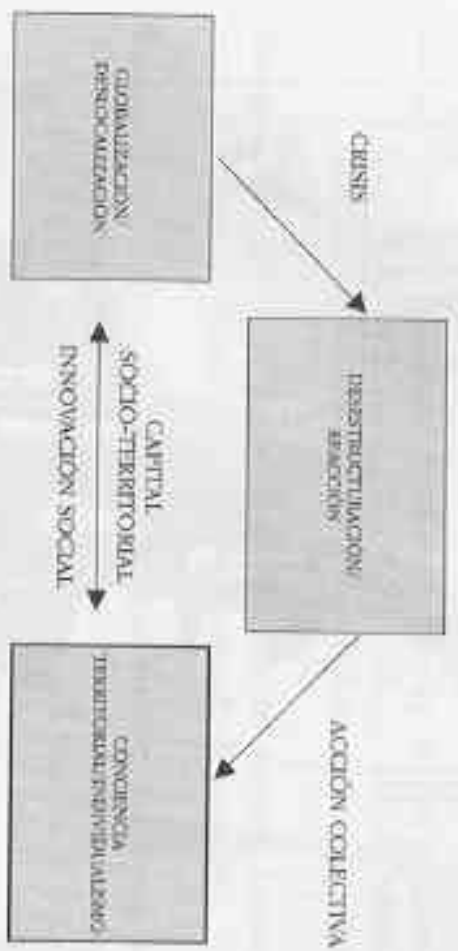




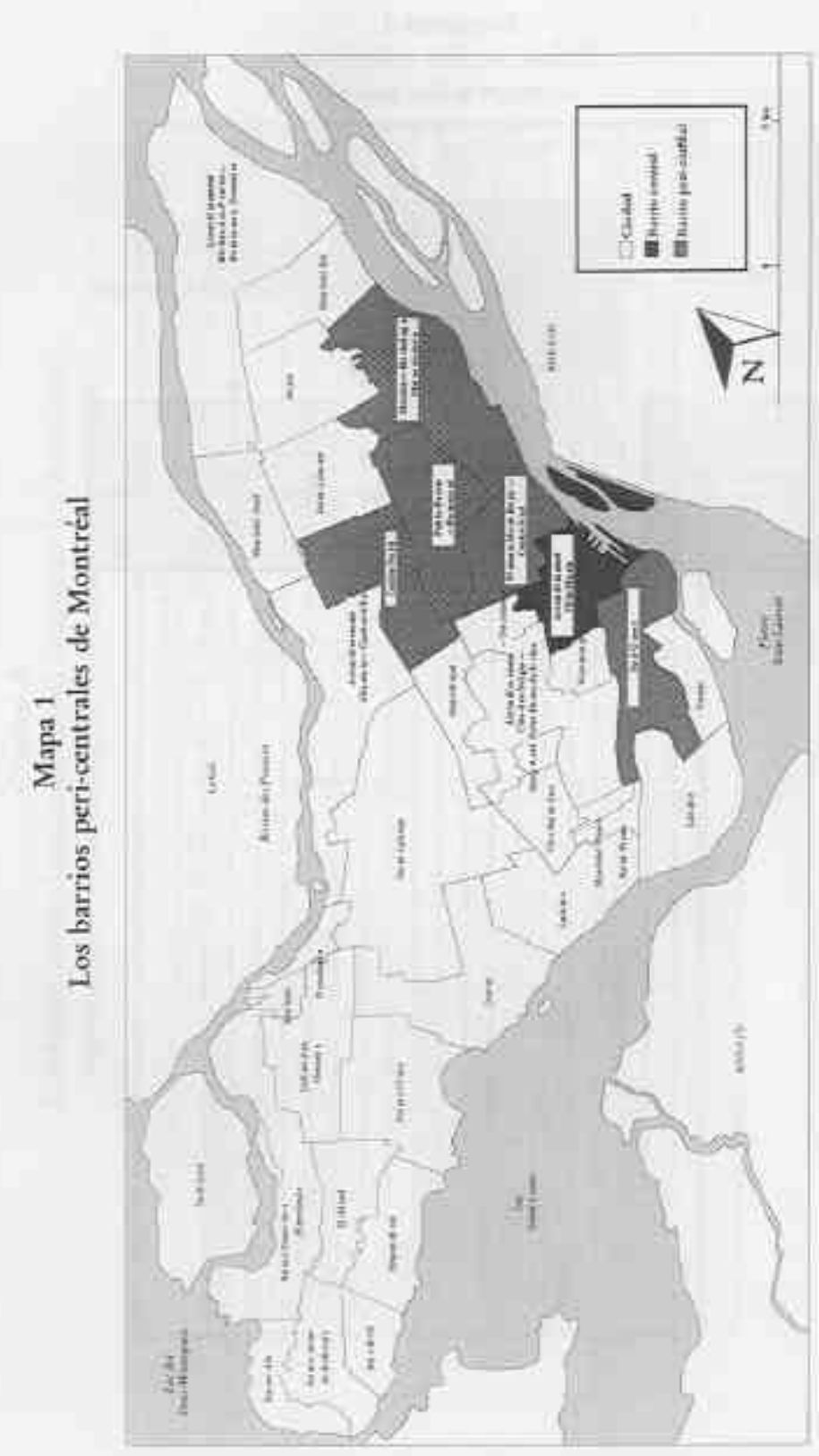

\section{Bibliografía}

Aydalot, Ph. (1984), “A la recherche des nouveaux dynamismes spatiaux", ent Aydalot, Ph. (ed.), Crise et espace, Paris, Economica, pp. 38.59.

(1986), "L'aptitude des milieuxlocaux d promouvoirl'innovation", en: Federwish, J, Zoller, H. G. (ed.), Technologie noucualle ef rapturnes régronales, Paris, Economica, P., $41-58$.

Bassand, M. (1997), Métropolisation er inegalitís sociales, Lausanne, Presses Polyrechniques et Universitaires Romandes:

Becattini, G., Rullani, E. (1995), "Systeme local et marche global le district industriel", en A. Rallet, Torre, A. (ed.), Economie industrielle et econome sputiale, París, Economica, pp. 171192

Beccatini, G. (1994), "El distrito marshalliano: una noción socioeconómica", en Benko, G., Lipietz, A. (ed.), Las regiones que sanan. Distritos y redes. Los mievos paradigmas de la geonrafta económica, Valencia, Edicions Alfons El Magnànim, Pp. $39-58$.

Benko, G., Lipietz, A. (1994) (ed.), Las regiones que gaman. Distritosy redes. Los muevos paradigmas de la geografia econónica, Valencia, Edicions Alfons El Magninim.

Coffey, W., Polese, M. (1993), "Le délin de lempire montrealais: regards sur l'économie d'une métropole en mutation", Recherches sociographiques, XXXIV, pp. 417-437.

- J, Droler, R. (1994), "La décentralisation intramétropolitaine des acrivités économiques dans la région de Montréal". Cahters de geographie dis Quéfes, 38 (105), pp. 371-394.

Crevoisier, O. (1994), "Dynamique industrielle et dynamique régionale: larticulation par les milieux innovareurs", Recue d'économie industrielle, to(4), PP. 33-48.

Favren1, L (1995), "Economie communautaire, mobilisation soctale et politiques publiques au Québec", en: Klein, J.-I., Levesque 
B. eds., Contre l'exclusion: repenser l'économie, Sainte-Foy, Presses de l'Université du Québec, pp. 175-186.

Favreau, L. et Ninacs, W. (1993), Pratiques de développement économique communautaire au Québec: de l'experimentation sociale à l'emergence d'une économie solidaire, Rapport de recherche, Santé et Bien-être Canada, Montréal, IFDEC.

Fitzgerald, J (1991), "Class and Community: the New Dynamics of Social Change", Environment and Planning D: Society and Space, 9, pp. 117-128.

Fontan, J.-M. (1991), Les corporations de développement économique communautaire montrealaises. Du développement économique communautaire au développement local de l'économie, Ph.D Disertation, Sociology, Université de Montréal, Montréal, IFDEQ.

(1994), "Le développement économique communautaire québecois: elements de synthèse et point de vue critique", Lien social et Politiques-RIAC, 32, pp. 115-126.

(1997), “L'État-région: une nouvelle forme de régulation des territoires?”, en: Coté, S., Klein, J.-L., Proulx, M.-U. (ed.), Action collective et décentralisation, Rimouski, GRIDEQ, PP. 233-254.

- Yaccarini, C. (1996), "Le projet Angus: une expérience novatrice de mobilisation locale au coeur de Montréal", Économie et solidarités, 28 (1), pp. 31-42.

Groupe Espace-Temps (1997), Bâtiments, entreprises, residences et espaces locatifs dans le Faubourg des Récollets en 1997, Montréal, Rapport présente à l'Agence Faubourg des Récollets.

Guay, L. (1997), "Le mouvement communautaire et l'économie sociale: entre l'espoir et le doute", en: Klein, J.-L., Tremblay, P.-A., Dionne, H. (ed.), Au delà du néoliberalisme: quel rôle pour les mouvements sociaux?, Québec, Presses de l'Université du Québec, pp. 135-154.
Hamel, P. (1991), Action collective et démocratielocale. Lesmouvements urbains Montréalais, Montréal, Presses de l'Université de Montréal.

(1995), “Mouvements urbains et modernité: l'exemple montréalais", Recherches sociographiques, XXXVI (2), pp. 279. 305.

(1997), "Démocratie pluraliste et action collective", en: Klein, J.-L., Tremblay, P.-A., Dionne, H. (ed.), $A u$ delà $d u$ néoliberalisme:quel rôle pourles mouvementssociaux?, Québec, Presses de l'Université du Québec, pp. 21-32.

Klein, J.-L. (1992), "Partenariat et planification flexible du developpement local", Revue canadiennedessciencesregionales. XV (3), pp. 491-505

(1997), “L'espace local à l'heure de la globalisation: la part de la mobilisation sociale", Cahiers de géographie du Québec, 41 (114), pp. 367-377.

Fontan, J.-M., Tremblay, D.-G., Tardif, C. (1998), “Les quartiers peri-centraux: le milieu communautaire dans la reconversion économique", en: Manzagol, C., Bryant, C. (ed.), Montréal 2001, Presses de l'Université de Montréal.

_ Tremblay, P.-A., Dionne, H. (1997) (ed.), $A u$ delà $d u$ néoliberalisme:quel rôle pourlesmouvementssociaux?, Québec, Presses de l'Université du Québec.

Waaub, J.-P. (1996), "Reconversion économique, développement local et mobilisation sociale: le cas de Montréal”, Recherchessociographiques, XXXVII (3), pp. 497-515

Maillat, D. (1992), "Milieux et dynamique territoriale de l'innovation", Revue canadienne des sciences régionales, 2 , XV(2), pp. 199-218.

Manzagol, C., Bryant, C. (1998) (ed), Montréal 2001, Montréal, Presses de l'Université de Montréal. 
Morin, R. (1995), “Communautés territoriales et insertion socioéconomique en milieu urbain”, en: Klein, J.-L. , Lévesque, B. eds. Contre, l'éxclusion: repenser l'économie, Sainte-Foy, Presses de l'Université du Québec, pp. 247-266.

- (1996), "Les corporations de développement économique communautaire et la relance des zones industrielles en déclin”, en: Tellier, L.-N. (ed.), Les défis et les options de la relance de Montréal, Québec, Presses de l'Université du Québec.

_ Latendresse, A., Parazelli, M. (1994), Les corporations de développement économique communautaire en milieu urbain: l'experience montréalaise, Montréal, Université du Québec à Montréal, Département d'Études urbaines et touristiques, Collection: Études, matériaux et documents, Num. 5.

Moulaert, F., Delladetsima, P. Leontidu, L. et al, (1994), Local development Strategies in Economically Desintegrated Areas: a Pro-Active Strategy Against Poverty in the European Community, Research ProgramEuropean Commission, Lille, Université de Lille 1, IFRESI- CNRS.

Delvainquière, J.-C., Delladetsima, P. (1997), “Les rapports sociaux dans le développement local: le rôle des mouvements sociaux", en: Klein, J.-L., Tremblay, P.-A., Dionne, H. (ed.), $A u$ delà du néoliberalisme: quel rôle pour les mouvements sociaux?, Québec, Presses de l'Université du Québec, pp. 77. 98.

Piore, M., Sabel, C.F. (1984), The Second IndustrialDivide. New York, Basic Books.

Proulx, M.-U. (1996), "Les relations économiques et politiques entre Montréal et les régions", en: Tellier, L.-N. (ed.), Les défiset les options de la relance de Montréal, Québec, Presses de l'Université du Québec.

Provost, S. (1997), La reconversion économique d'un ancien quartier industriel montréalais. L'évolution du sud du Faubourg des Récollets, Département de géographie. Activité de synthèse présentéé comme exigence pour la maîtrise en géographie, Non publiée.

Quevit, M. (1992), "Milieux innovateurs et couplage local-international dans les stratégies d'entreprises: cadre d'analyse", Revue canadienne des sciences regionales; $\mathrm{XV}(2)$, pp. 219-237.

Fisher, R., Kling, J. (1993) (ed.), Mobilizing the Community. Local Politics in the Era of the Global City, Newbury Park, CA, Sage Publications.

Remiggi, F., Senecal, G. (1992) (ed.), Montréal: Tableaux d'un espace en transformation, Montréal, ACFAS, Cahiers scientifiques.

Sénécal, G. Rose, D. Latouche, D. (1996), La relance du Faubourg des Récollets, Montréal, INRS-Urbanisation.

- Manzagol, C. (1993), "Montréal ou la métamorphose des territoires", Cahiers de géographie du Québec, 37 (101), pp. 351370.

Storper, M. (1997), "Les nouveaux dynamismes régionaux: conventions et systèmes d'acteurs", en: Cote, S., Klein, J.-L., Proulx, M.-U., (ed.), Action collective et décentralisation. Rimouski, GRIDEQ, pp. 1-17.

Tellier, L.-N. (1996) (ed.), Les défis et les options de la relance de Montréal. Québec, Presses de l'Université du Québec.

Thibodeau , J.-C. (1996), "La relance de Montréal: une question de long terme", en: Tellier, L.-N. (ed.), Les défis et les options de la relance de Montréal, Québec, Presses de l'Université du Québec, pp. 25-38.

Tremblay, D.-G. (1995), “L'aménagement et la réduction du temps de travail: une réponse au problème de l'exclusion?", en: Klein, J.-L., Lévesque, B. (ed.), Contrel'exclusion:repenserl'économie, Sainte-Foy, Presses de l'Université du Québec, pp. 99-121.

et Fontan, J.-M. (1994), Le développement économique local: la théorie, les pratiques, les expériences, Québec, Presses de l'Université du Québec. 